\title{
The Numbers of Thousand Place of Mersenne Primes*
}

\author{
Sibao Zhang ${ }^{1}$, Lihang Zhou ${ }^{2}$ \\ ${ }^{1}$ Department of Mathematics, Kashgar Teachers College, Kashgar, China \\ ${ }^{2}$ Department of Computer Science, Guangdong Technical College of Water Resources and \\ Electric Engineering, Guangzhou, China \\ E-mail: sibao98@sina.com
}

Received April 23, 2011; revised August 27, 2011; accepted September 4, 2011

\begin{abstract}
Mersenne primes are a special kind of primes, which are an important content in number theory. The study of Mersenne primes becomes one of hot topics of the nowadays science. Searching for Mersenne primes is very challenging in scientific researches. In this paper, the numbers of thousand place of Mersenne primes are studied, and the conclusion is presented by using the Chinese remainder theorem.
\end{abstract}

Keywords: Mersenne Primes, The Chinese Remainder Theorem, The Number of Thousand Place

\section{Introduction}

In $300 \mathrm{BC}$, ancient Greek mathematician Euclid proved that there are infinitude primes by contradiction, and raised that a small amount of prime numbers could be expressed in numbers of the form $2^{p}-1$, where $p$ is a prime. Afterward, many famous mathematicians have researched the prime numbers of this special formulation.

In 1644, French mathematician M. Mersenne stated in the preface to his Cogitata Physica-Mathematica that the numbers $2^{p}-1$ were primes for $p=2,3,5,7,13,17$, 19, 31, 67, 127 and 257, and were composite for all other positive integers $p<257$. Mersenne's (incorrect) conjecture fared only slightly better than Regius', but still got his name attached to these numbers. The formulation of $2^{p}-1$ is named as "Mersenne number" and expressed as $M_{p}$ (so $M_{p}=2^{p}-1$ ). If a $M_{p}$ is a prime number, then it is named as "Mersenne prime".

The Lucas-Lehmer test is a primarily test for Mersenne numbers: For $p$ an odd prime, the Mersenne number $2^{p}-1$ is prime if and only if $2^{p}-1$ divides $\mathrm{S}(p-1)$, where $\mathrm{S}(p+1)=\mathrm{S}(p)^{2}-2$, and $S(1)=4$. The test was originally developed by E. Lucas in 1856, and subsequently improved by Lucas in 1878 and D. Lehmer in the 1930s. The sequence $S(p)$ is computed modulo $2^{p}-1$ to save time. The Lucas-Lehmer test is ideal for binary computers because the division of $2^{p}-1$ (in binary) can be done by using rotation and addition only.

In 1992, Chinese mathematician and linguist Ha-

*Project Supported by the Science Foundation of Kashgar Teacher College (No. 112390). izhong Zhou [1] presented the well-known Zhou conjecture on the distribution of Mersenne primes in the natural number system: If $2^{2^{n}}<p<2^{2^{n+1}}(n=0,1,2,3, \cdots)$, then there are $2^{n+1}-1$ Mersenne primes. At the same time he gave the deduction: If $p<2^{2^{n+1}}$, then there are $2^{n+2}-n-2$ Mersenne primes.

As the increase of exponent $p$, searching for Mersenne primes is very challenging. Studying Mersenne primes, not only the advanced theory and practiced skills are needed, but also the arduous calculations are needed to validate whether a Mersenne number is a prime or not [2]. The research of Mersenne primes is very abundant in the contemporary theoretical and practical value. The research of Mersenne primes greatly promotes the development of mathematics, especially number theory. In addition, many well-known mathematics problems, such as Goldbach conjecture, twin primes, Riemann conjecture, etc. are inextricably linked to Mersenne primes. Therefore, the research of Mersenne primes can also speed up the solution to those problems [3]. And the research of Mersenne primes can promote distributed computing and programming arts. It not only requires well-designed distributed architecture, but also improves the numerical calculation methods and algorithm design arts. [4] There are only 47 known Mersenne primes [5].

In $[6,7]$, the last number and the ten place number have been studied, and conclusions have been obtained. In this paper, the numbers of thousand place of Mersenne primes are studied, and the conclusion is presented by using the Chinese remainder theorem.

Theorem If the exponent $p$ of $M_{p}$ satisfies 


$$
\left\{\begin{array}{l}
p=8 k+1, k \equiv 1,12,14,18,22,60 \\
61,63,66,69,75,103,104(\bmod 125) ; \\
p=8 k+3, k \equiv 14,27,46,54,61,63 \\
73,76,80,83,87,115(\bmod 125) ; \\
p=8 k+5, k \equiv 3,6,12,41,63,74 \\
76,84,122,123(\bmod 125) ; \\
p=8 k+7, k \equiv 0,10,13,17,20,24 \\
52,76,89,108,116,123(\bmod 125)
\end{array}\right.
$$

Then, the number of the thousand place of Mersenne primes is 0 ;

If the exponent $p$ of $M_{p}$ satisfies

$$
\left\{\begin{array}{l}
p=8 k+1, k \equiv 2,6,34,58,71,90,98 \\
105,107,117,120,124(\bmod 125) ; \\
p=8 k+3, k \equiv 16,17,19,22,25,31 \\
59,60,82,93,95,99,103(\bmod 125) ; \\
p=8 k+5, k \equiv 8,27,42,44,54,57 \\
61,64,68,96(\bmod 125) ; \\
p=8 k+7, k \equiv 19,30,32,36,40,78 \\
79,81,84,87,93,121,122(\bmod 125)
\end{array}\right.
$$

Then, the number of the thousand place of Mersenne primes is 1 ;

If the exponent $p$ of $M_{p}$ satisfies

$$
\left\{\begin{array}{l}
p=8 k+1, k \equiv 10,11,13,16,19,25 \\
53,54,76,87,89,93,97(\bmod 125) \\
p=8 k+3, k \equiv 1,5,8,12,40,64,77 \\
96,104,111,113,123(\bmod 125) \\
p=8 k+5, k \equiv 13,24,26,34 \\
72,73,78,81,87,116(\bmod 125) \\
p=8 k+7, k \equiv 1,14,33,41,48,50 \\
60,63,67,70,74,102(\bmod 125)
\end{array}\right.
$$

Then, the number of the thousand place of Mersenne primes is 2;

If the exponent $p$ of $M_{p}$ satisfies

$$
\left\{\begin{array}{l}
p=8 k+1, k \equiv 8,21,40,48,55,59 \\
67,70,74,77,81,109(\bmod 125) ; \\
p=8 k+3, k \equiv 7,18,20,24,28,66 \\
67,69,72,75,81,109,110(\bmod 125) ; \\
p=8 k+5, k \equiv 4,7,11,14,18,46 \\
83,102,117,119(\bmod 125) ; \\
p=8 k+7, k \equiv 3,4,6,9,12,18 \\
46,47,69,80,82,86,90(\bmod 125)
\end{array}\right.
$$

Then, the number of the thousand place of Mersenne primes is 3 ;
If the exponent $p$ of $M_{p}$ satisfies

$$
\left\{\begin{array}{l}
p=8 k+1, k \equiv 3,4,26,37,39,43,47 \\
85,86,88,91,94,100(\bmod 125) ; \\
p=8 k+3, k \equiv 2,21,29,36,38,48 \\
51,55,58,62,90,114(\bmod 125) ; \\
p=8 k+5, k \equiv 22,23,28,31,37 \\
66,88,99,101,109(\bmod 125) ; \\
p=8 k+7, k \equiv 27,51,64,83,91,98 \\
100,110,113,117,120,124(\bmod 125)
\end{array}\right.
$$

Then, the number of the thousand place of Mersenne primes is 4;

If the exponent $p$ of $M_{p}$ satisfies

$$
\left\{\begin{array}{l}
p=8 k+1, k \equiv 5,7,17,20,24,27,31 \\
59,83,96,115,123(\bmod 125) ; \\
p=8 k+3, k \equiv 0,6,34,35,57,68,70 \\
74,78,116,117,119,122(\bmod 125) ; \\
p=8 k+5, k \equiv 33,52,67,69,79 \\
82,86,89,93,121(\bmod 125) ; \\
p=8 k+7, k \equiv 5,7,11,15,53,54 \\
56,59,62,68,96,97,119(\bmod 125)
\end{array}\right.
$$

Then, the number of the thousand place of Mersenne primes is 5;

If the exponent $p$ of $M_{p}$ satisfies

$$
\left\{\begin{array}{l}
p=8 k+1, k \equiv 35,36,38,41,44,50,78 \\
79,101,112,114,118,122(\bmod 125) \\
p=8 k+3, k \equiv 15,39,52,71,79,86,88 \\
98,101,105,108,112(\bmod 125) \\
p=8 k+5, k \equiv 16,38,49,51,59,97 \\
98,103,106,112(\bmod 125) ; \\
p=8 k+7, k \equiv 8,16,23,25,35,38,42 \\
45,49,77,101,114(\bmod 125)
\end{array}\right.
$$

Then, the number of the thousand place of Mersenne primes is 6;

If the exponent $p$ of $M_{p}$ satisfies

$$
\left\{\begin{array}{l}
p=8 k+1, k \equiv 9,33,46,65,73,80 \\
82,92,95,99,102,106(\bmod 125) \\
p=8 k+3, k \equiv 3,41,42,44,47,50 \\
56,84,85,107,118,120,124(\bmod 125) \\
p=8 k+5, k \equiv 2,17,19,29,32,36 \\
39,43,71,108(\bmod 125) ; \\
p=8 k+7, k \equiv 21,22,44,55,57,61 \\
65,103,104,106,109,112,118(\bmod 125)
\end{array}\right.
$$

Then, the number of the thousand place of Mersenne primes is 7;

If the exponent $p$ of $M_{p}$ satisfies 


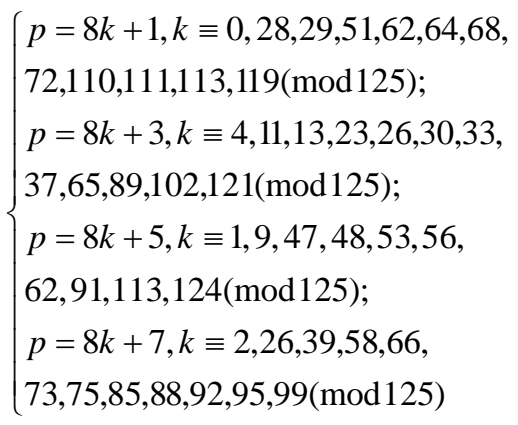

Then, the number of the thousand place of Mersenne primes is 8;

If the exponent $p$ of $M_{p}$ satisfies

$$
\left\{\begin{array}{l}
p=8 k+1, k \equiv 15,23,30,32,42,45 \\
49,52,56,84,108,121(\bmod 125) ; \\
p=8 k+3, k \equiv 9,10,32,43,45,49 \\
53,91,92,94,97,100,106(\bmod 125) ; \\
p=8 k+5, k \equiv 21,58,77,92,94,104 \\
107,111,114,118(\bmod 125) ; \\
p=8 k+7, k \equiv 28,29,31,34,37,43 \\
71,72,94,105,107,111,115(\bmod 125)
\end{array}\right.
$$

Then, the number of the thousand place of Mersenne primes is 9 .

\section{Preliminaries}

In order to prove the theorem, we need the following lemma.

\section{Lemma (The Chinese Remainder Theorem)}

Let $m_{1}, m_{2}, \cdots, m_{r}$ be pair-wise relatively prime positive integers. Then the system of congruencies

$$
\left\{\begin{array}{c}
x \equiv a_{1}\left(\bmod m_{1}\right) \\
x \equiv a_{2}\left(\bmod m_{2}\right) \\
\vdots \\
x \equiv a_{r}\left(\bmod m_{r}\right)
\end{array}\right.
$$

has a unique solution modulo $m=m_{1} m_{2}, \cdots, m_{r}$ [6].

\section{Proof of the Theorem}

Since the exponent $p$ of $M_{p}$ is a prime, then $p=4 k+1$ or $p=4 k+3$. When $p=2,3,5,2^{p}-1<100$. So, $p=8 k+$ 1 or $p=8 k+3$ or $p=8 k+5$ or $p=8 k+7$.

We have five congruence equations as following, when $M_{p}$ modulo 16,625 separately.

$$
\left\{\begin{array}{l}
M_{p}=2^{8 k+1}-1=256^{k} \times 2-1 \equiv-1(\bmod 16) \\
M_{p}=2^{8 k+3}-1=256^{k} \times 2^{3}-1 \equiv-1(\bmod 16) \\
M_{p}=2^{8 k+5}-1=256^{k} \times 2^{5}-1 \equiv-1(\bmod 16) \\
M_{p}=2^{8 k+7}-1=256^{k} \times 2^{7}-1 \equiv-1(\bmod 16)
\end{array}\right.
$$

$$
\begin{aligned}
& M_{p}=2^{8 k+1}-1 \equiv 256^{k} \times 2-1(\bmod 625) \\
& M_{p}=2^{8 k+3}-1 \equiv 256^{k} \times 2^{3}-1(\bmod 625) \\
& M_{p}=2^{8 k+5}-1 \equiv 256^{k} \times 2^{5}-1(\bmod 625) \\
& M_{p}=2^{8 k+7}-1 \equiv 256^{k} \times 2^{7}-1(\bmod 625)
\end{aligned}
$$

Note that $256^{k}=256^{125 k_{1}} \equiv 1(\bmod 625)$ when $k \equiv 0(\bmod 125)$, where $k$ is a nonnegative integer. Therefore, we have congruence equations as following, when $256^{k}$ modulo 625 separately, where $k \equiv k_{i}(\bmod 125)$ and $k_{i}=0,1,2, \cdots, 124$.

$256^{k} \equiv 1,256,536,341,421,276,31,436,366,571$, 551, 431, 336, 391, 96, 201, 206, 236, 416, 246, 476, 606, 136, 441, 396, 126, 381, 36, 466, 546, 401, 156, 561, 491, 71, 51, 556, 461, 516, 221, 326, 331, 361, 541, 371, 601, 106, 261, 566, 521, 251, 506, 161, 591, 46, 526, 281, 61, $616,196,176,56,586,16,346,451,456,486,41,496$, $101,231,386,66,21,376,6,286,91,171,26,406,186$, 116, 321, 301, 181, 86, 141, 471, 576, 581, 611, 166, 621, 226, 356, 511, 191, 146, 501, 131, 411, 216, 296, 151, 531, 311, 241, 446, 426, 306, 211, 266, 596, 76, 81, 111, 291, 121, 351, 481, 11, 316, $271(\bmod 625)$.

Combined congruence Equations (6) and (2), (3), (4), (5) separately, we have

$M_{p}=2^{8 k+1}-1 \equiv 1,511,446,56,216,551,61,246$, 106, 516, 476, 236, 46, 156, 191, 401, 411, 471, 206, 491, 326, 586, 271, 256, 166, 251, 136, 71, 306, 466, 176, 311, 496, 356, 141, 101, 486, 296, 406, 441, 26, 36, 96, 456, 116, 576, 211, 521, 506, 416, 501, 386, 321, 556, 91, 426, 561, 121, 606, 391, 351, 111, 546, 31, 66, 276, 286, 346, 81, 366, 201, 461, 146, 131, 41, 126, 11, 571, 181, 341, 51, 186, 371, 231, 16, 601, 361, 171, 281, 316, 526, 536, 596, 331, 616, 451, 86, 396, 381, 291, 376, 261, 196, 431, 591, 301, 436, 621, 481, 266, 226, 611, 421, 531, 566, 151, 161, 221, 581, 241, 76, 336, 21, 6, $541(\bmod 625)$.

$M_{p}=2^{8 k+3}-1 \equiv 7,172,537,227,242,332,247,362$, 427, 192, 32, 322, 187, 2, 142, 357, 397, 12, 202, 92, 57, 472, 462, 402, 42, 382, 547, 287, 602, 617, 82, 622, 112, 177, 567, 407, 72, 562, 377, 517, 107, 147, 387, 577, 467, 432, 222, 212, 152, 417, 132, 297, 37, 352, 367, 457, 372, 487, 552, 317, 157, 447, 312, 127, 267, 482, 522, 137, $327,217,182,597,587,527,167,507,47,412,102,117$, 207, 122, 237, 302, 67, 532, 197, 62, 502, 17, 232, 272, 512, 77, 592, 557, 347, 337, 277, 542, 257, 422, 162, 477, 492, 582, 497, 612, 52, 442, 282, 572, 437, 252, 392, 607, 22, 262, 452, 342, 307, 97, 87, 27, $292(\bmod 625)$.

$M_{p}=2^{8 k+5}-1 \equiv 31,66,276,286,346,81,366,201$, $461,146,131,41,126,11,571,181,341,51,186,371$, 
231, 16, 601, 361, 171, 281, 316, 526, 536, 596, 331, 616, 451, 86, 396, 381, 291, 376, 261, 196, 431, 591, 301, 436, 621, 481, 266, 226, 611, 421, 531, 566, 151, 161, 221, 581, 241, 76, 336, 21, 6, 541, 1,511, 446, 56, 216, 551, 61, 246, 106, 516, 476, 236, 46, 156, 191, 401, 411, 471, 206, 491, 326, 586, 271, 256, 166, 251, 136, 71, 306, 466, 176, 311, 496, 356, 141, 101, 486, 296, 406, 441, 26, 36, $96,456,116,576,211,521,506,416,501,386,321,556$, 91, 426, 561, 121, 606, 391, 351, 111, $546(\bmod 625)$.

$M_{p}=2^{8 k+7}-1 \equiv 127,267,482,522,137,327,217$, 182, 597, 587, 527, 167, 507, 47, 412, 102, 117, 207, 122, 237, 302, 67, 532, 197, 62, 502, 17, 232, 272, 512, 77, 592, 557, 347, 337, 277, 542, 257, 422, 162, 477, 492, 582, 497, 612, 52, 442, 282, 572, 437, 252, 392, 607, 22, 262, 452, 342, 307, 97, 87, 27, 292, 7, 172, 537, 227, 242, 332, 247, 362, 427, 192, 32, 322, 187, 2, 142, 357, 397, 12, 202, 92, 57, 472, 462, 402, 42, 382, 547, 287, 602, 617, 82, 622, 112, 177, 567, 407, 72, 562, 377, 517, 107, 147, 387, 577, 467, 432, 222, 212, 152, 417, 132, 297, 37, 352, 367, 457, 372, 487, 552, 317, 157, 447, $312(\bmod 625)$.

Combined congruence Equations (1) and (7), (8), (9), (10) separately, by using the Chinese Remainder Theorem, we have conclusions as following.

$M_{p}=2^{8 k+1}-1 \equiv 8751,511,1071,4431,4591,5551$, 1311, 5871, 3231, 7391, 2351, 2111, 671, 2031, 191, 9151, 2911, 5471, 831, 2991, 5951, 3711, 271, 9631, 5791, 2751, 4511, 5071, 8431, 8591, 9551, 5311, 9871, 7231, 1391, 6351, 6111, 4671, 6031, 4191, 3151, 6911, 9471, 4831, 6991, 9951, 7711, 4271, 3631, 9791, 6751, 8511, 9071, 2431, 2591, 3551, 9311, 3871, 1231, 5391, 351, 111, 8671, 31, 8191, 7151, 911, 3471, 8831, 991, 3951, 1711, 8271, 7631, 3791, 751, 2511, 3071, 6431, 6591, 7551, 3311, 7871, 5231, 9391, 4351, 4111, 2671, 4031, 2191, 1151, 4911, 7471, 2831, 4991, 7951, 5711, 2271, 1631, 7791, 4751, 6511, 7071, 431, 591, 1551, 7311, 1871, 9231, 3391, 8351, 8111, 6671, 8031, 6191, 5151, 8911, 1471, 6831, 8991, 1951, 9711, 6271, 5631, 1791( $\bmod 10000)$.

$M_{p}=2^{8 k+3}-1 \equiv 5007,2047,4287,7727,8367,2207$, 5247, 3487, 2927, 9567, 9407, 8447, 2687, 8127, 767, 6607, 1647, 1887, 3327, 1967, 3807, 4847, 1087, 8527, 3167, 1007, 8047, 287, 3727, 4367, 8207, 1247, 9487, 8927, 5567, 5407, 4447, 8687, 4127, 6767, 2607, 7647, 7887, 9327, 7967, 9807, 847, 7087, 4527, 9167, 7007, 4047, 6287, 9727, 367, 4207, 7247, 5487, 4927, 1567, 1407, 447, 4687, 127, 2767, 8607, 3647, 3887, 5327, 3967, 5807, 6847, 3087, 527, 5727, 3007, 47, 2287, 5727, 6367, 207, 3247, 1487, 927, 7567, 7407, 6447, 687, 6127, 8767, 4607, 9647, 9887, 1327, 9967, 1807, 2847, 9087,
6527, 1167, 9007, 6047, 8287, 1727, 2367, 6207, 9247, 7487, 6927, 3567, 3407, 2447, 6687, 2127, 4767, 607, 5647, 5887, 7327, 5967, 7807, 8847, 5087, 2527, $7167(\bmod 10000)$.

$M_{p}=2^{8 k+5}-1 \equiv 31,8191,7151,911,3471,8831,991$, 3951, 1711, 8271, 7631, 3791, 751, 2511, 3071, 6431, 6591, 7551, 3311, 7871, 5231, 9391, 4351, 4111, 2671, 4031, 2191, 1151, 4911, 7471, 2831, 4991, 7951, 5711, 2271, 1631, 7791, 4751, 6511, 7071, 431, 591, 1551, 7311, 1871, 9231, 3391, 8351, 8111, 6671, 8031, 6191, 5151, 8911, 1471, 6831, 8991, 1951, 9711, 6271, 5631, 1791, 8751, 511, 1071, 4431, 4591, 5551, 1311, 5871, 3231, 7391, 2911, 2111, 671, 2031, 191, 9151, 2911, 5471, 831, 2991, 5951, 3711, 271, 9631, 5791, 2751, 4511, 5071, 8431, 8591, 9551, 5311, 9871, 7231, 1391, 6351, 6111, 4671, 6031, 4191, 3151, 6911, 9471, 4831, 6991, 9951, 7711, 4271, 3631, 9791, 6751, 8511, 9071, 2431, 2591, 3551, 9311, 3871, 1231, 5391, 351, 111, 8671( $\bmod 10000)$.

$M_{p}=2^{8 k+7}-1 \equiv 127,2767,8607,3647,3887,5327$, 3967, 5807, 6847, 3087, 527, 5167, 3007, 47, 2287, 5727, 6367, 207, 3247, 1487, 927, 7567, 7407, 6447, 687, 6127, 8767, 4607, 9647, 9887, 1327, 9967, 1807, 2847, 9087, 6527, 1167, 9007, 6047, 8287, 1727, 2367, 6207, 9247, 7487, 6927, 3567, 3407, 2447, 6687, 2127, 4767, 607, 5647, 5887, 7327, 5967, 7807, 8847, 5087, 2527, 7167, 5007, 2047, 4287, 7727, 8367, 2207, 5247, 3487, 2927, 9567, 9407, 8447, 2687, 8127, 767, 6607, 1647, 1887, 3327, 1967, 3807, 4847, 1087, 8527, 3167, 1007, 8047, 287, 3727, 4367, 8207, 1247, 9487, 8927, 5567, 5407, 4447, 8687, 4127, 6767, 2607, 7647, 7887, 9327, 7967, 9807, 847, 7087, 4527, 9167, 7007, 4047, 6287, 9727, 367, 4207, 7247, 5487, 4927, 1567, 1407, 447, 4687( $\bmod 10000)$.

From the congruence Equations (11)-(14), if $k \equiv 0,5$, $10,15,20,25,30,35,40,45,50,55,60,65,70,75,80$, $85,90,95,100,105,110,115,120(\bmod 125)$, then $p=8 k+5$ are composites, and then these numbers are not considered. So we can draw the conclusion. That completes the proof.

\section{Acknowledgements}

The authors wish to thank Dr. Alden More for many helpful discussions and comments on the manuscript.

\section{References}

[1] H. Z. Zhou, "The Distribution of Mersenne Primes," in Chinese, Acta Scientiarum Naturalium Universitatis Sunyatseni, Vol. 31, No. 4, 1992, pp. 121-122. 
[2] S. B. Zhang, X. C. Ma and L. H. Zhou, "Some Notes on the Distribution of Mersenne Primes," Applied Mathematics, Vol. 1, No. 4, 2010, pp. 312-315. doi:10.4236/am.2010.14041

[3] Q. Cheng and L. Feng, "The Research of Mersenne Primes Based on Distributed Computing," Frontier Science, Vol. 4, No. 14, 2010, pp. 59-67.

[4] S. B. Zhang, "Review of Research on Mersenne Primes," Science \& Technology Review, Vol. 26, No. 18, 2008, pp. 88-92.

[5] Q. Chen and P. Zhang, “A Math Treasure: Mersenne
Primes,” in Chinese, Encyclopedic Knowledge, Vol. 32, No. 5, 2009, p. 22.

[6] S. B. Zhang and A. Yunus, "A Note on Mersenne Numbers," in Chinese, Journal of Hebei North University, Vol. 26, No. 2, 2010, pp. 11-12.

[7] S. B. Zhang, "The Mantissa of Mersenne Primes," in Chinese, Journal of Jilin Normal University, Vol. 31, No. 2, 2010, pp. 92-94.

[8] K. H. Rosen, "Elementary Number Theory and It's Applications,” Pearson Education, Inc., Upper Saddle River, 2005. 